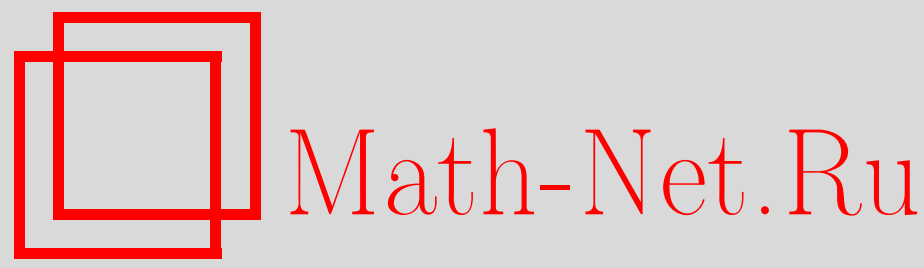

Д. Н. Запорожец, А. И. Назаров, Как мало бывает корней у случайного полинома в среднем?, Теория вероятн. и ее примен., 2008, том 53, выпуск 1, 40-58

DOI: https://doi.org/10.4213/tvp318

Использование Общероссийского математического портала MathNet.Ru подразумевает, что вы прочитали и согласны с пользовательским соглашением

http://www . mathnet.ru/rus/agreement

Параметры загрузки:

IP : 3.85 .183 .62

26 апреля 2023 г., 14:47:04

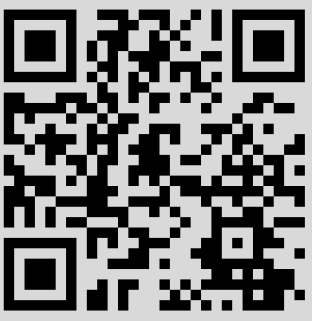




\section{КАК МАЛО БЫВАЕТ КОРНЕЙ У СЛУЧАЙНОГО ПОЛИНОМА В СРЕДНЕМ? ${ }^{1)}$}

Пусть $\mathscr{N}\left(G_{n}\right)$ означает число вещественных корней случайного полинома $G_{n}$ степени $n$ с независимыми одинаково распределенными случайными коэффициентами. В работе изучается вопрос, насколько малой может быть величина $\sup _{n \in \mathbf{N}} \mathbf{E} \mathscr{N}\left(\mathbf{G}_{\mathbf{n}}\right)$ при разных условиях, наложенных на распределение коэффициентов $G_{n}$.

Ключевые слова и фразы: случайный полином, среднее число вещественных корней.

Введение. Пусть $\Theta$ - множество всех невырожденных (т.е. не сводящихся к одному атому) вероятностных распределений на $\mathbf{R}$. Возьмем последовательность $\left\{\xi_{n}\right\}_{n=0}^{\infty}$ независимых случайных величин с распределением $\theta \in \Theta$ и рассмотрим полиномы одной переменной

$$
G_{n}(t)=\xi_{n} t^{n}+\xi_{n-1} t^{n-1}+\cdots+\xi_{1} t+\xi_{0}, \quad n \in \mathbf{N} .
$$

Обозначим $\mathscr{N}\left(G_{n}\right)$ число вещественных корней полинома $G_{n}$, посчитанных с учетом их кратности. Что можно сказать о среднем значении $\mathscr{N}\left(G_{n}\right)$ ?

Первые содержательные асимптотические оценки $\mathbf{E}_{\theta} \mathscr{N}\left(G_{n}\right)$ при $n \rightarrow \infty$ для некоторых конкретных распределений $\theta$ были получены в работах [1]-[4]. Вскоре после этого М. Кац [5] получил первый асимптотически точный результат. Он доказал справедливость соотношения

$$
\mathbf{E} \mathscr{N}\left(G_{n}\right)=\frac{2}{\pi} \log n \cdot(1+o(1)), \quad n \rightarrow \infty,
$$

для стандартньх гауссовских коэффициентов (см. также [6], [7] для других конкретных распределений $\xi_{0}$ ). Впоследствии И.А. Ибрагимов и

* С.-Петербургское отделение Математического института им. В. А. Стеклова PAН, ул. Фонтанка, 27, 191023 С.-Петербург, Россия; e-mail: zap1979@gmail.com

** С.-Петербургский государственный университет, математико-механический факультет, Старый Петергоф, Университетский пр-т, 28, 198504 С.-Петербург, Россия; e-mail: an@an4751.spb.edu

1) Работа выполнена при поддержке РФФИ (гранты 07-01-00159, 08-01-00098) и программы «Ведущие научные школы» (грант НШ-4222.2006.1). 
Н. Б. Маслова [8] обобщили этот результат на класс распределений, принадлежащих области притяжения произвольного невырожденного устойчивого закона (коэффициент $2 / \pi$ при этом заменяется на константу, зависящую от параметров устойчивого закона).

Несмотря на большую общность данного результата, сушествуют распределения, дающие принципиально другое поведение среднего числа вещественных корней полинома. Так, в [9] было построено невырожденное симметричное распределение коэффициентов, при котором среднее число вещественных корней $G_{n}$ ограничено равномерно по $n \in \mathrm{N}$ (а именно, $\left.\mathrm{E} \mathscr{N}\left(G_{n}\right)<9\right)$. Возникает естественный вопрос: насколько можно улучшить оценку 9 в этом утверждении? Ответ на него дает следующая теорема.

Теорема 1. Для $a, b \geqslant 0$ таких, что $0<a+b \leqslant 1$, обозначим $\Theta_{a, b} \subset \Theta$ множество всех распределений случайной величинь $\xi_{0}$, для которых $\mathbf{P}\left\{\xi_{0}>0\right\}=a, \mathbf{P}\left\{\xi_{0}<0\right\}=b$. Справедливо следуюшее соотношение:

$$
\inf _{\theta \in \Theta_{a, b}} \sup _{n \in \mathbb{N}} \mathbf{E}_{\theta}\left\{\mathscr{N}\left(G_{n}\right) \mid G_{n}(t) \not \equiv 0\right\}=1+\frac{1-|a-b|}{a+b} .
$$

Статья посвящена доказательству теоремы 1 и организована следующим образом. В п. 1 доказываются несколько вспомогательных утверждений о полиномах. В п. 2 сначала выводятся асимптотические оценки вероятностей некоторых событий, которые потом используются для оценки $\mathbf{E} \mathscr{N}\left(G_{n}\right)$ снизу. Для оценки сверху в п. 3 применяется модификация конструкции из [9]. Совпадение верхней и нижней оценок и доказывает теорему. Пункт 4 содержит несколько простых следствий.

Наряду с обозначением $\mathscr{N}$ мы будем использовать $\mathscr{N}^{+}$и $\mathscr{N}^{-}$ число положительных и отрицательных корней соответственно. Также положим $c \stackrel{\text { def }}{=} \mathbf{P}\left\{\xi_{0}=0\right\}=1-a-b$.

\section{1. Вспомогательные утверждения о полиномах.}

Лемма 1. Пусть дан полином $g(t)=a_{m} t^{m}+\cdots+a_{1} t+a_{0}, a_{0} a_{m} \neq 0$. 1. $\mathscr{N}^{+}(g)$ оченивается снизу следуюшим образом:

\begin{tabular}{|l|c|c|}
\hline & $a_{0} g(1)>0$ & $a_{0} g(1)<0$ \\
\hline$a_{0} a_{m}>0$ & $\mathscr{N}^{+}(g) \geqslant 0$ & $\mathscr{N}^{+}(g) \geqslant 2$ \\
\hline$a_{0} a_{m}<0$ & \multicolumn{2}{|c|}{$\mathscr{N}^{+}(g) \geqslant 1$} \\
\hline
\end{tabular}

2. $\mathscr{N}^{-}(g)$ оченивается снизу следуюшим образом:

\begin{tabular}{|c|c|c|}
\hline & $a_{0} g(-1)>0$ & $a_{0} g(-1)<0$ \\
\hline$a_{0} a_{m}>0$ & $\mathscr{N}^{-}(g) \geqslant \frac{1}{2}\left[1-(-1)^{m}\right]$ & $\mathscr{N}^{-}(g) \geqslant 1+\frac{1}{2}\left[1+(-1)^{m}\right]$ \\
\hline$a_{0} a_{m}<0$ & $\mathscr{N}^{-}(g) \geqslant \frac{1}{2}\left[1+(-1)^{m}\right]$ & $\mathscr{N}^{-}(g) \geqslant 1+\frac{1}{2}\left[1-(-1)^{m}\right]$ \\
\hline
\end{tabular}


Д о к а з а т е л ь с т в о. Первое утверждение непосредственно вытекает из теоремы Коши о промежуточном значении непрерывной функции. Второе утверждение получается из первого заменой $t \rightarrow-t$.

Лемма 2. Пусть дан полином $g(t)=a_{m} t^{m}+\cdots+a_{1} t+a_{0}$, причем $\left|a_{m}\right|,\left|a_{0}\right| \geqslant 1$ и $a_{m} a_{0}>0$. Если существует коэффичиент $a_{k}$ такой, что $a_{k} a_{0}>0 u$

$$
\left|a_{k}\right| \geqslant\left(m \max _{i \neq k}\left\{\left|a_{i}\right|\right\}\right)^{2 m}
$$

то полином $g(t)$ не имеет положительньх корней.

Д ок аз ат ель с т в о. Введем обозначение $K \stackrel{\text { def }}{=} \max _{i \neq k}\left\{\left|a_{i}\right|\right\}$. Очевидно, что $K \geqslant 1$. Представим $g(t)$ в следующем виде:

$$
g(t)=\left(a_{m} t^{m}+a_{k} t^{k}+a_{0}\right)+\sum_{i \neq 0, k, m} a_{i} t^{i}
$$

(здесь мы предположили, что $k \neq 0, m$; случаи равенства разбираются совершенно аналогично). Для того чтобы доказать, что $g$ не имеет положительных корней, достаточно проверить, что при $t>0$ выражение в скобках в правой части (2) строго больше оставшейся суммы по абсолютной величине. Действительно, при $0<t \leqslant(m K)^{-1}$ выполнено

$$
\left|a_{m} t^{m}+a_{k} t^{k}+a_{0}\right|>\left|a_{0}\right| \geqslant 1 \geqslant m \cdot K t \geqslant \sum_{i \neq 0, k, m}\left|a_{i}\right| t \geqslant\left|\sum_{i \neq 0, k, m} a_{i} t^{i}\right|
$$

при $(m K)^{-1} \leqslant t \leqslant m K$ выполнено

$$
\begin{aligned}
\left|a_{m} t^{m}+a_{k} t^{k}+a_{0}\right| & >\left|a_{k} t^{k}\right| \geqslant(m K)^{2 m}(m K)^{-k} \\
& \geqslant m \cdot K(m K)^{m-1} \geqslant\left|\sum_{i \neq 0, k, m} a_{i} t^{i}\right| ;
\end{aligned}
$$

при $t \geqslant m K$ выполнено

$$
\left|a_{m} t^{m}+a_{k} t^{k}+a_{0}\right|>\left|a_{m} t^{m}\right| \geqslant t^{m} \geqslant m \cdot K t^{m-1} \geqslant\left|\sum_{i \neq 0, k, m} a_{i} t^{i}\right| .
$$

Лемма 3. Пусть дан полином $g(t)=a_{m} t^{m}+\cdots+a_{1} t+a_{0}, a_{0} a_{m} \neq 0$, c коэффициентами, среди которых есть как положительные, так и отрицательные. Пусть $l$ есть минимальный индекс, для которого выполнено $a_{0} a_{l}<0$. Тогда

$$
\mathscr{N}^{+}(g) \leqslant \mathscr{N}^{+}\left(g^{(l)}\right)+1
$$

Д ок а з а т е л с т в о. Для удобства изложения будем считать, что $a_{0}>0, a_{l}<0$. Пусть $s$ обозначает максимальный индекс такой, что $s<l$ и $a_{s} \neq 0$ (тем самым, $a_{s}>0$ ). По теореме Ролля

$$
\mathscr{N}^{+}\left(g^{(s)}\right) \leqslant \mathscr{N}^{+}\left(g^{(s+1)}\right)+1 \text {. }
$$


Далее, для любого $i=0, \ldots, s-1$ у полинома $g^{(i)}$ свободный член неотрицателен, а самый младший из ненулевых коэффициентов, за исключением свободного члена, положителен. Поэтому, как легко видеть, между минимальным положительным корнем $g^{(i)}$ (если он есть) и началом координат найдется по меньшей мере один корень $g^{(i+1)}$. Это выполняется также и при $i=s+1, \ldots, l-1$, так как в этом случае в начале координат лежит корень $g^{(i)}$. Следовательно, по теореме Ролля выполнено

$$
\mathscr{N}^{+}\left(g^{(i)}\right) \leqslant \mathscr{N}^{+}\left(g^{(i+1)}\right), \quad i=0, \ldots, s-1, s+1, \ldots, l-1 .
$$

Объединение неравенств (3) и (4) доказывает лемму.

Лемма 4. Пусть дан полином $g(t)=a_{m} t^{m}+\cdots+a_{1} t+a_{0}$, $a_{0} a_{m} \neq 0$, причем все ненулевье коэффициенты удовлетворяют неравенству $\left|a_{i}\right| \geqslant 1$. Если существует коэффициент $a_{k}$, для которого

$$
\left|a_{k}\right| \geqslant\left((m !)^{m} \max _{i \neq k}\left\{\left|a_{i}\right|\right\}\right)^{2 m}
$$

то количество положительньх корней полинома $g(t)$ оченивается сверху следуюшим образом:

\begin{tabular}{|c|c|c|}
\hline & $a_{0} a_{k}>0$ & $a_{0} a_{k}<0$ \\
\hline$a_{0} a_{m}>0$ & $\mathscr{N}^{+}(g)=0$ & $\mathscr{N}^{+}(g) \leqslant 2$ \\
\hline$a_{0} a_{m}<0$ & \multicolumn{2}{|c|}{$\mathscr{N}^{+}(g) \leqslant 1$} \\
\hline
\end{tabular}

Д ок а з а т е л с т в о. При $a_{0} a_{m}>0, a_{0} a_{k}>0$ отсутствие положительных корней непосредственно следует из леммы 2.

Пусть $a_{0} a_{m}<0$. Можно считать, что $a_{0} a_{k}<0$ : в противном случае надо вместо $g(t)$ рассмотреть полином

$$
\widetilde{g}(t) \stackrel{\text { def }}{=} a_{0} t^{m}+\cdots+a_{m-1} t+a_{m},
$$

после чего воспользоваться очевидным равенством $\mathscr{N}^{+}(g)=\mathscr{N}^{+}(\tilde{g})$. Обозначим $l$ минимальный индекс, для которого $a_{0} a_{l}<0$. Тогда $1 \leqslant l \leqslant k$ и

$$
g^{(l)}(t)=\sum_{i=0}^{m-l} \frac{(l+i) !}{i !} a_{l+i} t^{i}
$$

При $i \neq k-l$ имеем

$$
\left((m-l) \frac{(l+i) !}{i !}\left|a_{l+i}\right|\right)^{2(m-l)} \leqslant\left((m !)^{m}\left|a_{l+i}\right|\right)^{2 m} \leqslant\left|a_{k}\right| \leqslant \frac{k !}{(k-l) !}\left|a_{k}\right|
$$

следовательно, $g^{(l)}$ удовлетворяет условию леммы 2, и $\mathscr{N}^{+}\left(g^{(l)}\right)=0$. По лемме 3 получаем $\mathscr{N}^{+}(g) \leqslant \mathscr{N}^{+}\left(g^{(l)}\right)+1=1$. 
Пусть, наконец, $a_{0} a_{m}>0, a_{0} a_{k}<0$. Снова воспользовавшись введенным обозначением $l$, при $i \neq k-l$ имеем

$$
\left(((m-l) !)^{m-l} \frac{(l+i) !}{i !}\left|a_{l+i}\right|\right)^{2(m-l)} \leqslant\left((m !)^{m}\left|a_{l+i}\right|\right)^{2 m} \leqslant\left|a_{k}\right| \leqslant \frac{k !}{(k-l) !}\left|a_{k}\right|
$$

следовательно, $g^{(l)}$ удовлетворяет условию настоящей леммы и подпадает под предыдущий, уже доказанный, случай, поэтому $\mathscr{N}^{+}\left(g^{(l)}\right) \leqslant 1$. По лемме 3 получаем $\mathscr{N}^{+}(g) \leqslant \mathscr{N}^{+}\left(g^{(l)}\right)+1 \leqslant 2$.

Следствие. $B$ условиях леммы 4 количество вещественных корней полинома оченивается следуюшим образом:

\begin{tabular}{|c|c|c|}
\hline & $a_{0} a_{k}>0$ & $a_{0} a_{k}<0$ \\
\hline$a_{0} a_{m}>0$ & $\mathscr{N}(g) \leqslant 1-\frac{1}{2}(-1)^{k}\left[1+(-1)^{m}\right]$ & $\mathscr{N}(g) \leqslant 3+\frac{1}{2}(-1)^{k}\left[1+(-1)^{m}\right]$ \\
\hline$a_{0} a_{m}<0$ & $\mathscr{N}(g) \leqslant 2-\frac{1}{2}(-1)^{k}\left[1-(-1)^{m}\right]$ & $\mathscr{N}(g) \leqslant 2+\frac{1}{2}(-1)^{k}\left[1-(-1)^{m}\right]$ \\
\hline
\end{tabular}

Д о к а з а т е л ь с т в о. Применяя лемму 4 к полиному $G(-t)$, получаем оценку числа отрицательных корней:

\begin{tabular}{|c|c|c|}
\hline & $a_{0} a_{k}>0$ & $a_{0} a_{k}<0$ \\
\hline$a_{0} a_{m}>0$ & $\mathscr{N}^{-}(g) \leqslant 1-\frac{1}{2}(-1)^{k}\left[1+(-1)^{m}\right]$ & $\mathscr{N}^{-}(g) \leqslant 1+\frac{1}{2}(-1)^{k}\left[1+(-1)^{m}\right]$ \\
\hline$a_{0} a_{m}<0$ & $\mathscr{N}^{-}(g) \leqslant 1-\frac{1}{2}(-1)^{k}\left[1-(-1)^{m}\right]$ & $\mathscr{N}^{-}(g) \leqslant 1+\frac{1}{2}(-1)^{k}\left[1-(-1)^{m}\right]$ \\
\hline
\end{tabular}

Осталось ее объединить с оценкой числа положительных корней, полученной в лемме (нулевых корней нет, так как $a_{0} \neq 0$ ).

2. Оценка снизу. Сначала получим асимптотические оценки вероятностей некоторых событий, которые потом будут нами использованы для вывода оценки величины $\mathbf{E} \mathscr{N}\left(G_{n}\right)$ снизу. Для этого нам понадобятся следующие обозначения:

$$
S_{n}^{+} \stackrel{\text { def }}{=} \sum_{i=1}^{n} \xi_{i}=G_{n}(1)-G_{n}(0), \quad S_{n}^{-} \stackrel{\text { def }}{=} \sum_{i=1}^{n}(-1)^{i} \xi_{i}=G_{n}(-1)-G_{n}(0) .
$$

Лемма 5. Для любого $A>0$ выполнено

$$
\begin{aligned}
& \lim _{n \rightarrow \infty} \mathbf{P}\left\{\left|S_{n}^{-}\right|<A\right\}=0 ; \\
& \lim _{n \rightarrow \infty} \mathbf{P}\left\{\left|S_{n}^{+}\right|<A\right\}=0 .
\end{aligned}
$$

Д ок а зател ь с т в. Данные соотношения следуют из того, что при сложении независимых случайных величин функция концентрации не возрастает, а функция концентрации произвольного невырожденного случайного блуждания стремится к нулю (см., например, [10, гл. 4]). 
Лемма 6. Выполнень следующие соотношения:

$$
\begin{aligned}
& \lim _{n \rightarrow \infty} \mathbf{P}\left\{\xi_{0}\left(\xi_{0}+S_{n-1}^{-}\right) \leqslant 0, \xi_{0}\left(\xi_{0}+S_{n}^{-}\right)>0\right\}=0 ; \\
& \lim _{n \rightarrow \infty} \mathbf{P}\left\{\xi_{0} S_{n-1}^{+} \leqslant 0, \xi_{0}\left(\xi_{0}+S_{n}^{+}\right) \geqslant 0\right\}=0 .
\end{aligned}
$$

Д о к а з а т е л ь с т в о. Докажем (8). Для этого зафиксируем $\varepsilon>0$ и подберем $A>0$ такое, что $\mathbf{P}\left\{\left|\xi_{0}\right|+\left|\xi_{n}\right| \geqslant A\right\}<\varepsilon$. Получаем

$$
\begin{aligned}
& \mathbf{P}\left\{\xi_{0}\left(\xi_{0}+S_{n-1}^{-}\right) \leqslant 0, \xi_{0}\left(\xi_{0}+S_{n}^{-}\right)>0\right\} \leqslant \mathbf{P}\left\{\left|S_{n-1}^{-}\right| \leqslant\left|\xi_{0}\right|+\left|\xi_{n}\right|\right\} \\
&= \mathbf{P}\left\{\left|S_{n-1}^{-}\right| \leqslant\left|\xi_{0}\right|+\left|\xi_{n}\right||| \xi_{0}|+| \xi_{n} \mid \geqslant A\right\} \mathbf{P}\left\{\left|\xi_{0}\right|+\left|\xi_{n}\right| \geqslant A\right\} \\
&+\mathbf{P}\left\{\left|S_{n-1}^{-}\right| \leqslant\left|\xi_{0}\right|+\left|\xi_{n}\right||| \xi_{0}|+| \xi_{n} \mid<A\right\} \mathbf{P}\left\{\left|\xi_{0}\right|+\left|\xi_{n}\right|<A\right\} \\
& \leqslant+\mathbf{P}\left\{\left|S_{n-1}^{-}\right|<A\right\} .
\end{aligned}
$$

По лемме 5 второе слагаемое стремится к нулю при $n \rightarrow \infty$, поэтому, в силу произвольности $\varepsilon,(8)$ доказано. Соотношение (9) доказывается аналогично с использованием (7).

Лемма 7. Выполнены следуюшие соотношения:

$$
\begin{aligned}
& \liminf _{n \rightarrow \infty} \mathbf{P}\left\{\xi_{0} \xi_{n}>0, \xi_{0}\left(\xi_{0}+S_{n}^{+}\right)<0\right\} \\
& \left.\quad \geqslant \frac{1}{2}\left[\mathbf{P}\left\{\xi_{0}>0\right\}\right]^{2}+\left[\mathbf{P}\left\{\xi_{0}<0\right\}\right]^{2}-\left|\left[\mathbf{P}\left\{\xi_{0}>0\right\}\right]^{2}-\left[\mathbf{P}\left\{\xi_{0}<0\right\}\right]^{2}\right|\right) ; \\
& \lim _{n \rightarrow \infty} \mathbf{P}\left\{\xi_{n} \neq 0, \xi_{0}\left(\xi_{0}+S_{n}^{-}\right)>0\right\} \\
& \quad=\lim _{n \rightarrow \infty} \mathbf{P}\left\{\xi_{n} \neq 0, \xi_{0}\left(\xi_{0}+S_{n}^{-}\right)<0\right\}=\frac{1}{2} \mathbf{P}\left\{\xi_{0} \xi_{n} \neq 0\right\} ; \\
& \lim _{n \rightarrow \infty} \mathbf{P}\left\{\xi_{0} \neq 0, \xi_{n}\left(\xi_{0}+S_{n}^{-}\right)>0\right\} \\
& \quad=\lim _{n \rightarrow \infty} \mathbf{P}\left\{\xi_{0} \neq 0, \xi_{n}\left(\xi_{0}+S_{n}^{-}\right)<0\right\}=\frac{1}{2} \mathbf{P}\left\{\xi_{0} \xi_{n} \neq 0\right\} .
\end{aligned}
$$

Д о к а з а т е л ь с т в о. Для доказательства неравенства (10) зафиксируем произвольное $\varepsilon>0$. Используя (9), получаем, что при всех достаточно больших $n$ выполнено

$$
\begin{aligned}
\mathbf{P}\left\{\xi_{0} \xi_{n}>0, \xi_{0}\left(\xi_{0}+S_{n}^{+}\right)<0\right\} \geqslant \mathbf{P}\left\{\xi_{0} \xi_{n}>0, \xi_{0} S_{n-1}^{+} \leqslant 0\right\} \\
\quad-\mathbf{P}\left\{\xi_{0} \xi_{n}>0, \xi_{0} S_{n-1}^{+} \leqslant 0, \xi_{0}\left(\xi_{0}+S_{n}^{+}\right) \geqslant 0\right\} \\
\geqslant \mathbf{P}\left\{\xi_{0} \xi_{n}>0, \xi_{0} S_{n-1}^{+} \leqslant 0\right\}-\varepsilon
\end{aligned}
$$

Далее,

$$
\begin{aligned}
\mathbf{P} & \left\{\xi_{0} \xi_{n}>0, \xi_{0} S_{n-1}^{+} \leqslant 0\right\} \\
& =\mathbf{P}\left\{\xi_{0}>0, \xi_{n}>0, S_{n-1}^{+} \leqslant 0\right\}+\mathbf{P}\left\{\xi_{0}<0, \xi_{n}<0, S_{n-1}^{+} \geqslant 0\right\} \\
& =\left[\mathbf{P}\left\{\xi_{0}>0\right\}\right]^{2} \mathbf{P}\left\{S_{n-1}^{+} \leqslant 0\right\}+\left[\mathbf{P}\left\{\xi_{0}<0\right\}\right]^{2} \mathbf{P}\left\{S_{n-1}^{+} \geqslant 0\right\} \\
& \geqslant \frac{1}{2}\left(\left[\mathbf{P}\left\{\xi_{0}>0\right\}\right]^{2}+\left[\mathbf{P}\left\{\xi_{0}<0\right\}\right]^{2}-\left|\left[\mathbf{P}\left\{\xi_{0}>0\right\}\right]^{2}-\left[\mathbf{P}\left\{\xi_{0}<0\right\}\right]^{2}\right|\right),
\end{aligned}
$$

таким образом, (10) доказано. 
Перейдем к доказательству соотношений (11). Заметим, что, в силу (8) и независимости $\xi_{n}$ от $\xi_{0}$ и $S_{n-1}^{-}$, соотношения (11) равносильны следующим:

$$
\lim _{n \rightarrow \infty} \mathbf{P}\left\{\xi_{0}\left(\xi_{0}+S_{n}^{-}\right)>0\right\}=\lim _{n \rightarrow \infty} \mathbf{P}\left\{\xi_{0}\left(\xi_{0}+S_{n}^{-}\right)<0\right\}=\frac{1}{2} \mathbf{P}\left\{\xi_{0} \neq 0\right\} .
$$

Для доказательства (13) зафиксируем произвольное $\varepsilon>0$ и подберем такое $B>0$, для которого выполнено $\mathbf{P}\left\{\left|\xi_{0}\right|<B\right\}>1-\varepsilon$. Если $n$ четно, то $S_{n}^{-}$является симметрично распределенной случайной величиной. Поэтому в силу (6) существует такое $N$, что при четном $n \geqslant N-1$ выполнено $\mathbf{P}\left\{S_{n}^{-}>2 B\right\}>1 / 2-\varepsilon$. Если же $n$ нечетно, то, ввиду четности $n-1$, при $n \geqslant N$ получаем

$$
\begin{aligned}
\mathbf{P}\left\{S_{n}^{-}>B\right\} & =\mathbf{P}\left\{S_{n-1}^{-}-\xi_{n}>B\right\} \geqslant \mathbf{P}\left\{S_{n-1}^{-}>2 B, \xi_{n}<B\right\} \\
& \geqslant \mathbf{P}\left\{S_{n-1}^{-}>2 B\right\}-\mathbf{P}\left\{\xi_{n} \geqslant B\right\} \geqslant \frac{1}{2}-2 \varepsilon .
\end{aligned}
$$

В результате при любом $n \geqslant N$ имеем

$$
\mathbf{P}\left\{\xi_{0}>-B, S_{n}^{-}>B\right\} \geqslant \mathbf{P}\left\{S_{n}^{-}>B\right\}-\mathbf{P}\left\{\xi_{0} \leqslant-B\right\} \geqslant \frac{1}{2}-3 \varepsilon .
$$

Следовательно,

$$
\begin{aligned}
\mathbf{P} & \left\{\xi_{0}<0, \xi_{0}+S_{n}^{-}>0\right\} \\
& \geqslant \mathbf{P}\left\{\xi_{0}<0, \xi_{0}+S_{n}^{-}>0 \mid \xi_{0}>-B, S_{n}^{-}>B\right\} \mathbf{P}\left\{\xi_{0}>-B, S_{n}^{-}>B\right\} \\
& \geqslant\left(\frac{1}{2}-3 \varepsilon\right) \mathbf{P}\left\{\xi_{0}<0, \xi_{0}+S_{n}^{-}>0 \mid \xi_{0}>-B, S_{n}^{-}>B\right\} \\
& =\left(\frac{1}{2}-3 \varepsilon\right) \mathbf{P}\left\{\xi_{0}<0 \mid \xi_{0}>-B, S_{n}^{-}>B\right\} .
\end{aligned}
$$

В силу независимости $\xi_{0}$ и $S_{n}^{-}$,

$$
\mathbf{P}\left\{\xi_{0}<0 \mid \xi_{0}>-B, S_{n}^{-}>B\right\}=\mathbf{P}\left\{\xi_{0}<0 \mid \xi_{0}>-B\right\},
$$

поэтому

$$
\begin{aligned}
\mathbf{P}\left\{\xi_{0}<0, \xi_{0}+S_{n}^{-}>0\right\} & \geqslant\left(\frac{1}{2}-3 \varepsilon\right) \mathbf{P}\left\{\xi_{0}<0 \mid \xi_{0}>-B\right\} \\
& \geqslant\left(\frac{1}{2}-3 \varepsilon\right)\left(\mathbf{P}\left\{\xi_{0}<0\right\}-\varepsilon\right) .
\end{aligned}
$$

Точно так же доказывается, что при всех достаточно больших $n$

$$
\mathbf{P}\left\{\xi_{0}>0, \xi_{0}+S_{n}^{-}<0\right\} \geqslant\left(\frac{1}{2}-3 \varepsilon\right)\left(\mathbf{P}\left\{\xi_{0}>0\right\}-\varepsilon\right) .
$$

Складывая эти два неравенства и учитывая произвольность $\varepsilon>0$, получаем

$$
\liminf _{n \rightarrow \infty} \mathbf{P}\left\{\xi_{0}\left(\xi_{0}+S_{n}^{-}\right)<0\right\} \geqslant \frac{1}{2} \mathbf{P}\left\{\xi_{0} \neq 0\right\} .
$$


Аналогично получается соотношение

$$
\liminf _{n \rightarrow \infty} \mathbf{P}\left\{\xi_{0}\left(\xi_{0}+S_{n}^{-}\right)>0\right\} \geqslant \frac{1}{2} \mathbf{P}\left\{\xi_{0} \neq 0\right\} .
$$

Осталось заметить, что

$$
\mathbf{P}\left\{\xi_{0}\left(\xi_{0}+S_{n}^{-}\right)>0\right\}+\mathbf{P}\left\{\xi_{0}\left(\xi_{0}+S_{n}^{-}\right)<0\right\} \leqslant \mathbf{P}\left\{\xi_{0} \neq 0\right\} .
$$

Последние три неравенства доказывают (13) и, следовательно, (11). Соотношение (12) доказывается аналогично.

Теперь перейдем к доказательству основного утверждения этого пункта.

Лемма 8. Для любого распределения $\theta \in \Theta_{a, b}$ выполнено

$$
\sup _{n \in \mathbf{N}} \mathbf{E}_{\theta}\left\{\mathscr{N}\left(G_{n}\right) \mid G_{n}(t) \not \equiv 0\right\} \geqslant 1+\frac{1-|a-b|}{a+b} .
$$

Д о к а з а т е л ь с т в о. Зафиксируем $\varepsilon>0$ и выберем, в соответствии с леммой 7 , такое $N$, что при $n \geqslant N$ выполнены соотношения

$$
\mathbf{P}\left\{\xi_{0} \xi_{n}>0, \xi_{0} G_{n}(1)<0\right\} \geqslant \frac{1}{2}\left(a^{2}+b^{2}-\left|a^{2}-b^{2}\right|\right)-\varepsilon
$$

И

$$
\begin{aligned}
& \left|(a+b)^{-2} \mathbf{P}\left\{\xi_{n} \neq 0, \xi_{0} G_{n}(-1)>0\right\}-\frac{1}{2}\right|<\varepsilon, \\
& \left|(a+b)^{-2} \mathbf{P}\left\{\xi_{n} \neq 0, \xi_{0} G_{n}(-1)<0\right\}-\frac{1}{2}\right|<\varepsilon, \\
& \left|(a+b)^{-2} \mathbf{P}\left\{\xi_{0} \neq 0, \xi_{n} G_{n}(-1)>0\right\}-\frac{1}{2}\right|<\varepsilon, \\
& \left|(a+b)^{-2} \mathbf{P}\left\{\xi_{0} \neq 0, \xi_{n} G_{n}(-1)<0\right\}-\frac{1}{2}\right|<\varepsilon .
\end{aligned}
$$

Оценим сначала среднее число корней полинома $G_{n}$ в предположении, что $\xi_{0} \xi_{n} \neq 0$. Для положительной полуоси, в соответствии с первой таблицей из леммы 1 , при $n \geqslant N$ получаем:

$$
\begin{aligned}
& \mathbf{E}\left\{\mathscr{N}^{+}\left(G_{n}\right) \mid \xi_{0} \xi_{n} \neq 0\right\}=\left[\mathbf{P}\left\{\xi_{0} \xi_{n} \neq 0\right\}\right]^{-1} \\
& \times\left(\mathbf{E}\left\{\mathscr{N}^{+}\left(G_{n}\right) \mid \xi_{0} \xi_{n}>0, \xi_{0} G_{n}(1) \geqslant 0\right\} \mathbf{P}\left\{\xi_{0} \xi_{n}>0, \xi_{0} G_{n}(1) \geqslant 0\right\}\right. \\
& \quad+\mathbf{E}\left\{\mathscr{N}^{+}\left(G_{n}\right) \mid \xi_{0} \xi_{n}>0, \xi_{0} G_{n}(1)<0\right\} \mathbf{P}\left\{\xi_{0} \xi_{n}>0, \xi_{0} G_{n}(1)<0\right\} \\
& \left.\quad+\mathbf{E}\left\{\mathscr{N}^{+}\left(G_{n}\right) \mid \xi_{0} \xi_{n}<0\right\} \mathbf{P}\left\{\xi_{0} \xi_{n}<0\right\}\right) \\
& \geqslant(a+b)^{-2}\left(2 \mathbf{P}\left\{\xi_{0} \xi_{n}>0, \xi_{0} G_{n}(1)<0\right\}+1 \cdot \mathbf{P}\left\{\xi_{0} \xi_{n}<0\right\}\right) .
\end{aligned}
$$

Применение (15) приводит к неравенствам

$$
\begin{aligned}
\mathrm{E}\left\{\mathscr{N}^{+}\left(G_{n}\right) \mid \xi_{0} \xi_{n} \neq 0\right\} & \geqslant(a+b)^{-2}\left(a^{2}+b^{2}-\left|a^{2}-b^{2}\right|-2 \varepsilon+2 a b\right) \\
& \geqslant 1-\frac{|a-b|}{a+b}-\frac{2 \varepsilon}{(a+b)^{2}} .
\end{aligned}
$$


Для отрицательной полуоси, в соответствии со второй таблицей из леммы 1 , при $n \geqslant N$ получаем:

$$
\begin{aligned}
& \mathbf{E}\left\{\mathscr{N}^{-}\left(G_{n}\right) \mid \xi_{0} \xi_{n} \neq 0\right\} \geqslant\left[\mathbf{P}\left\{\xi_{0} \xi_{n} \neq 0\right\}\right]^{-1} \\
& \times\left(\mathbf{E}\left\{\mathscr{N}^{-}\left(G_{n}\right) \mid \xi_{0} \xi_{n}>0, \xi_{0} G_{n}(-1)>0\right\} \cdot \mathbf{P}\left\{\xi_{0} \xi_{n}>0, \xi_{0} G_{n}(1)>0\right\}\right. \\
& +\mathbf{E}\left\{\mathscr{N}^{-}\left(G_{n}\right) \mid \xi_{0} \xi_{n}>0, \xi_{0} G_{n}(-1)<0\right\} \cdot \mathbf{P}\left\{\xi_{0} \xi_{n}>0, \xi_{0} G_{n}(1)<0\right\} \\
& +\mathbf{E}\left\{\mathscr{N}^{-}\left(G_{n}\right) \mid \xi_{0} \xi_{n}<0, \xi_{0} G_{n}(-1)>0\right\} \cdot \mathbf{P}\left\{\xi_{0} \xi_{n}<0, \xi_{0} G_{n}(1)>0\right\} \\
& \left.+\mathbf{E}\left\{\mathscr{N}^{-}\left(G_{n}\right) \mid \xi_{0} \xi_{n}<0, \xi_{0} G_{n}(-1)<0\right\} \cdot \mathbf{P}\left\{\xi_{0} \xi_{n}<0, \xi_{0} G_{n}(1)<0\right\}\right) \\
& \geqslant(a+b)^{-2}\left(\frac{1}{2}\left[1-(-1)^{n}\right] \mathbf{P}\left\{\xi_{0} \xi_{n}>0, \xi_{0} G_{n}(1)>0\right\}\right. \\
& +\left[1+\frac{1}{2}\left[1+(-1)^{n}\right]\right] \mathbf{P}\left\{\xi_{0} \xi_{n}>0, \xi_{0} G_{n}(1)<0\right\} \\
& +\frac{1}{2}\left[1+(-1)^{n}\right] \mathbf{P}\left\{\xi_{0} \xi_{n}<0, \xi_{0} G_{n}(1)>0\right\} \\
& \left.+\left[1+\frac{1}{2}\left[1-(-1)^{n}\right]\right] \mathbf{P}\left\{\xi_{0} \xi_{n}<0, \xi_{0} G_{n}(1)<0\right\}\right) \\
& =(a+b)^{-2}\left(\frac{1}{2} \mathbf{P}\left\{\xi_{n} \neq 0, \xi_{0} G_{n}(-1)>0\right\}+\frac{3}{2} \mathbf{P}\left\{\xi_{n} \neq 0, \xi_{0} G_{n}(-1)<0\right\}\right. \\
& \left.+(-1)^{n}\left[\mathbf{P}\left\{\xi_{0} \neq 0, \xi_{n} G_{n}(-1)<0\right\}-\mathbf{P}\left\{\xi_{0} \neq 0, \xi_{n} G_{n}(-1)>0\right\}\right]\right) .
\end{aligned}
$$

Применение (16) приводит к неравенству

$$
\mathbf{E}\left\{\mathscr{N}^{-}\left(G_{n}\right) \mid \xi_{0} \xi_{n} \neq 0\right\} \geqslant 1-4 \varepsilon
$$

Откажемся теперь от предположения $\xi_{0} \xi_{n} \neq 0$. Рассмотрим случайные величины

$$
\zeta_{n} \stackrel{\text { def }}{=} \min \left\{i=0, \ldots, n \mid \xi_{n-i} \neq 0\right\}, \quad \eta_{n} \stackrel{\text { def }}{=} \min \left\{j=0, \ldots, n \mid \xi_{j} \neq 0\right\} .
$$

Поскольку при $\eta_{n}=j$ полином $G_{n}$ имеет нулевой корень кратности $j$, при $n \geqslant N$ и $M=\{0 \leqslant i, j \leqslant(n-N) / 2\}$ получаем

$$
\begin{aligned}
\mathbf{E}\{ & \left.\mathscr{N}\left(G_{n}\right) \mid G_{n}(t) \not \equiv 0\right\} \\
& \geqslant \sum_{(i, j) \in M} \mathbf{E}\left\{\mathscr{N}\left(G_{n}\right) \mid \zeta_{n}=i, \eta_{n}=j\right\} \mathbf{P}\left\{\zeta_{n}=i, \eta_{n}=j\right\} \\
& =\sum_{(i, j) \in M}\left(\mathbf{E}\left\{\mathscr{N}^{-}\left(G_{n-i-j}\right)+\mathscr{N}^{+}\left(G_{n-i-j}\right) \mid \xi_{0} \xi_{n-i-j} \neq 0\right\}+j\right) \\
& \geqslant\left(1-\frac{|a-b|}{a+b}-\frac{2 \varepsilon}{(a+b)^{2}}+1-4 \varepsilon\right) \sum_{(i, j) \in M} c^{i+j}(1-c)^{2}
\end{aligned}
$$


Поскольку при $n \rightarrow \infty$

$$
\begin{aligned}
& \sum_{(i, j) \in M} c^{i+j}(1-c)^{2} \longrightarrow 1 \\
& \sum_{(i, j) \in M} j c^{i+j}(1-c)^{2} \longrightarrow \frac{c}{1-c}=\frac{1}{a+b}-1
\end{aligned}
$$

ввиду произвольности $\varepsilon$ приходим к (14).

3. Оценка сверху. Для получения оценки сверху мы будем пользоваться результатами леммы 4, поэтому нашей первой задачей является построение такого распределения коэффициентов, для которого с большой вероятностью полином $G_{n}(t)$ будет удовлетворять ее условиям.

Лемма 9. Если $a \geqslant b$, то для произвольного $\delta>0$ существует распределение коэффичиентов $\theta_{\delta} \in \Theta_{a, b}$ такое, что при всех $n$ случайный полином $G_{n}$ обладает следующими двумя свойствами:

$$
\mathbf{P}_{\theta_{\delta}}\left\{R_{n} \mid G_{n}(t) \not \equiv 0\right\} \geqslant 1-\delta n^{-1},
$$

где событие $R_{n}$ означает, что $G_{n}$ удовлетворяет условиям леммы 4;

$$
\mathbf{P}_{\theta_{\delta}}\left\{R_{n}^{\prime} \mid A_{n}\right\} \geqslant 1-\delta n^{-1},
$$

где собьтие $R_{n}^{\prime}$ означает, что у $G_{n}$ максимальный по модулю коэфФиииент положителен, а событие $A_{n}$ - что у $G_{n}$ есть по крайней мере один положительный коэффиииент.

Д о к а з а т е л ь с т в о. Для построения $\theta_{\delta} \in \Theta_{a, b}$ мы модифицируем идею из [9]. Рассмотрим вероятностное распределение с дискретным носителем:

$$
\mathbf{P}\left\{\xi_{0}=q_{k}\right\}=a p_{k}, \quad \mathbf{P}\left\{\xi_{0}=-r_{k}\right\}=b p_{k}, \quad k \in \mathbf{N},
$$

где $\sum_{k=1}^{\infty} p_{k}=1,0<q_{1}<\cdots<q_{k}<\cdots<\infty, 0<r_{1}<\cdots<r_{k}<\cdots$ $<\infty$, и

$$
\mathbf{P}\left\{\xi_{0}=0\right\}=c=1-a-b .
$$

Рассмотрим последовательность независимых одинаково распределенных случайных величин $\xi_{1}^{\prime}, \ldots, \xi_{n}^{\prime}, \ldots$ с распределением $\mathbf{P}\left\{\xi_{1}^{\prime}=q_{k}\right\}=$ $p_{k}, k \in \mathrm{N}$, а также последовательность независимых одинаково распределенных случайных величин $\xi_{1}^{\prime \prime}, \ldots, \xi_{n}^{\prime \prime}, \ldots$ с распределением $\mathbf{P}\left\{\xi_{1}^{\prime \prime}=r_{k}\right\}=$ $p_{k}, k \in \mathbf{N}$. Пусть $\mu=\mu(n)$ обозначает число положительньх коэффициентов у $G_{n}$, а $\nu=\nu(n)$ - число отрицательньг. Легко видеть, что положительные коэффициенты распределены, как $\xi_{i}^{\prime}$, а отрицательные как $\left(-\xi_{i}^{\prime \prime}\right)$. Так как для вычисления среднего числа корней нам важны не сами значения коэффициентов, а их распределение, в дальнейшем мы будем считать, что $\xi_{1}^{\prime}, \ldots, \xi_{\mu}^{\prime}$ суть положительные коэффициенты полинома $G_{n}$, а $\left(-\xi_{1}^{\prime \prime}\right), \ldots,\left(-\xi_{\nu}^{\prime \prime}\right)$ - отрицательные (их порядок нам будет не важен). 
Для произвольных независимых одинаково распределенных случайных величин $\tilde{\xi}_{1}, \ldots, \tilde{\xi}_{m}$ с некоторым счетным положительным носителем $\left\{s_{1}, \ldots, s_{k}, \ldots\right\}\left(0<s_{1}<\cdots<s_{k}<\cdots<\infty\right)$ введем в рассмотрение событие $Q\left(\tilde{\xi}_{1}, \ldots, \tilde{\xi}_{m}\right)$, заключающееся в том, что среди этих случайных величин существует ровно одна максимальная, причем она равна некоторому $s_{l}$ c $l \geqslant m$. В [9] показано, что для любого $\varepsilon>0$ точкам носителя можно приписать такие вероятности, что при всех $m$ будет выполнено

$$
\mathbf{P}\left\{Q\left(\tilde{\xi}_{1}, \ldots, \tilde{\xi}_{m}\right)\right\}>1-\varepsilon m^{-2} .
$$

Следовательно, мы можем задать последовательность $\left\{p_{k}\right\}$ таким образом, что при всех $m$ будет выполняться неравенство

$$
\mathbf{P}\left\{Q\left(\xi_{1}^{\prime}, \ldots, \xi_{m}^{\prime}\right)\right\}=\mathbf{P}\left\{Q\left(\xi_{1}^{\prime \prime}, \ldots, \xi_{m}^{\prime \prime}\right)\right\}>1-\varepsilon m^{-2} .
$$

Рассмотрим строго возрастающую последовательность $l(k) \in \mathbf{N}$ и некоторое $N \in \mathbf{N}$. Положим $N_{k} \stackrel{\text { def }}{=} \max \left\{(k+1)^{2}, N\right\}$ и зададим $r_{k}, q_{k}$ следующим образом:

$$
r_{k+1}=\left[\left(N_{k} !\right)^{N_{k}+1 / 2} r_{k}\right]^{4 N_{k}^{2}}, \quad r_{1}=1 ; \quad q_{k}=\left[\left(N_{l(k)} !\right)^{N_{l(k)}} r_{l(k)}\right]^{2 N_{l(k)}} .
$$

Покажем, что при подходящем выборе $\{l(k)\}, N$ и $\varepsilon$ будут выполняться свойства (19), (20).

Предложение 1. Предположим, что $a \geqslant b>0$. Тогда существует $N=N(a, b, \delta)$ такое, ито при $n \geqslant N$ выполнено

$$
\mathbf{P}\{\mu, \nu \geqslant[\sqrt{n}]+1\} \geqslant 1-\frac{\delta}{2 n} .
$$

Доказательство предложения 1. Имеем:

$$
\begin{aligned}
1 & -\mathbf{P}\{\mu, \nu \geqslant[\sqrt{n}]+1\} \leqslant \mathbf{P}\{\mu \leqslant[\sqrt{n}]\}+\mathbf{P}\{\nu \leqslant[\sqrt{n}]\} \\
& =\sum_{i=0}^{[\sqrt{n}]} a^{i}(b+c)^{n+1-i} \frac{(n+1) !}{i !(n+1-i) !}+\sum_{j=0}^{[\sqrt{n}]} b^{j}(a+c)^{n+1-j} \frac{(n+1) !}{j !(n+1-j) !} \\
& \leqslant 2 \sum_{i=0}^{[\sqrt{n}]}(1-b)^{n+1} \frac{(n+1) !}{i !(n+1-i) !} \leqslant 2([\sqrt{n}]+1)(1-b)^{n+1}(n+1)^{[\sqrt{n}]} .
\end{aligned}
$$

Осталось заметить, что при $n \rightarrow \infty$ выполнено

$$
\left[2([\sqrt{n}]+1)(1-b)^{n+1}(n+1)^{[\sqrt{n}]}\right] n \rightarrow 0 .
$$

Предложение 1 доказано.

Вернемся к доказательству леммы. Будем считать, что $b>0$ (случай $b=0$ разбирается еще проще). Зафиксируем такое $N$, что при $n \geqslant N$ выполнено (23). Сначала подберем такое $\varepsilon$, при котором выполняется (19), а после этого так построим $\{l(k)\}$, чтобы выполнялось $(20)$.

Определим событие $R_{n}^{*}$ следующим образом:

$$
R_{n}^{*} \stackrel{\text { def }}{=} \begin{cases}Q\left(\xi_{1}^{\prime}, \ldots, \xi_{\mu}^{\prime}\right) \cap Q\left(\xi_{1}^{\prime \prime}, \ldots, \xi_{\nu}^{\prime \prime}\right), & \text { если } n<N, \\ Q\left(\xi_{1}^{\prime}, \ldots, \xi_{\mu}^{\prime}\right) \cap Q\left(\xi_{1}^{\prime \prime}, \ldots, \xi_{\nu}^{\prime \prime}\right) \cap\{\mu, \nu \geqslant[\sqrt{n}]+1\}, & \text { если } n \geqslant N,\end{cases}
$$


где в случае $\mu=0$ под $Q\left(\xi_{1}^{\prime}, \ldots, \xi_{\mu}^{\prime}\right)$ мы подразумеваем достоверное событие (аналогично для $\nu$ ).

В силу (21), при $n<N$ имеем

$$
\mathbf{P}\left\{R_{n}^{*} \mid G_{n} \not \equiv 0\right\} \geqslant 1-2 \varepsilon,
$$

а при $n \geqslant N$, учитывая также и (23), получаем

$$
\begin{aligned}
\mathbf{P}\left\{R_{n}^{*} \mid G_{n} \neq \equiv 0\right\}= & \mathbf{P}\left\{Q\left(\xi_{1}^{\prime}, \ldots, \xi_{\mu}^{\prime}\right) \cap Q\left(\xi_{1}^{\prime \prime}, \ldots, \xi_{\nu}^{\prime \prime}\right) \mid \mu, \nu \geqslant[\sqrt{n}]+1\right\} \\
& \times \mathbf{P}\left\{\mu, \nu \geqslant[\sqrt{n}]+1 \mid G_{n} \not \equiv 0\right\} \\
\geqslant & \left(1-\frac{2 \varepsilon}{([\sqrt{n}]+1)^{2}}\right) \mathbf{P}\{\mu, \nu \geqslant[\sqrt{n}]+1\} \\
\geqslant & \left(1-\frac{2 \varepsilon}{([\sqrt{n}]+1)^{2}}\right)\left(1-\frac{\delta}{2 n}\right) \geqslant 1-\frac{2 \varepsilon}{n}-\frac{\delta}{2 n} .
\end{aligned}
$$

Если взять $\varepsilon \leqslant \min \{\delta /(2 N), \delta / 4\}$, то, учитывая (24) и $(25)$, для выполнения (19) осталось доказать, что $R_{n}^{*} \subset R_{n}$.

Пусть произошло событие $R_{n}^{*}$. Будем считать, что $\mu, \nu>0$ (случай, когда все коэффициенты либо неотрицательные, либо неположительные, разбирается еще проще). Тогда существуют ровно один максимальный положительный коэффициент и ровно один минимальный отрицательный, равные некоторым $q_{m^{\prime}}$ и $\left(-r_{m^{\prime \prime}}\right)$, причем $N_{m^{\prime}}, N_{m^{\prime \prime}} \geqslant n$.

Применяя (22), нетрудно проверить, что в случае $m^{\prime \prime}<l\left(m^{\prime}\right)+1$ условию (5) удовлетворяет коэффициент $q_{m^{\prime}}$, а в случае $m^{\prime \prime} \geqslant l\left(m^{\prime}\right)+1$ коэффициент $\left(-r_{m^{\prime \prime}}\right)$, следовательно, условие леммы 4 выполнено.

Перейдем к заданию значений $\{l(k)\}$. При $b=0$ выполнено $\mathbf{P}\left\{R_{n}^{\prime} \mid\right.$ $\left.A_{n}\right\}=1$, поэтому можно считать, что $b>0$. Если мы выберем настолько большое $l(1)$, что

$$
\mathbf{P}\left\{\max \left(\xi_{1}^{\prime \prime}, \ldots, \xi_{N}^{\prime \prime}\right)<q_{1}\right\} \geqslant 1-\varepsilon
$$

то при $n<N$ получим

$$
\begin{aligned}
\mathbf{P}\left\{R_{n}^{\prime} \mid A_{n}\right\} & \geqslant \mathbf{P}\left\{\max \left(\xi_{1}^{\prime \prime}, \ldots, \xi_{N}^{\prime \prime}\right)<\xi_{1}^{\prime}\right\} \\
& \geqslant \mathbf{P}\left\{\max \left(\xi_{1}^{\prime \prime}, \ldots, \xi_{N}^{\prime \prime}\right)<q_{1}\right\} \geqslant 1-\varepsilon .
\end{aligned}
$$

Пусть теперь $n \geqslant N$. Выберем последовательность $\{l(k)\}$ настолько быстро растущей, чтобы было выполнено неравенство

$$
\mathbf{P}\left\{\max \left(\xi_{1}^{\prime \prime}, \ldots, \xi_{k^{2}}^{\prime \prime}\right)<q_{k}\right\} \geqslant 1-\varepsilon k^{-1}, \quad k \in \mathbf{N} .
$$

Тогда при $i \geqslant[\sqrt{n}]+1, j \leqslant n$ будет верна оценка

$$
\begin{aligned}
\mathbf{P} & \left\{\max \left(\xi_{1}^{\prime}, \ldots, \xi_{i}^{\prime}\right)>\max \left(\xi_{1}^{\prime \prime}, \ldots, \xi_{j}^{\prime \prime}\right)\right\} \\
& \geqslant \mathbf{P}\left\{\max \left(\xi_{1}^{\prime}, \ldots, \xi_{[\sqrt{n}]+1}^{\prime}\right)>\max \left(\xi_{1}^{\prime \prime}, \ldots, \xi_{n}^{\prime \prime}\right)\right\} \\
& \geqslant \mathbf{P}\left\{\max \left(\xi_{1}^{\prime}, \ldots, \xi_{[\sqrt{n}]+1}^{\prime}\right) \geqslant q_{[\sqrt{n}]+1}, \max \left(\xi_{1}^{\prime \prime}, \ldots, \xi_{n}^{\prime \prime}\right)<q_{[\sqrt{n}]+1}\right\} \\
& \geqslant \mathbf{P}\left\{Q\left(\xi_{1}^{\prime}, \ldots, \xi_{[\sqrt{n}]+1}^{\prime}\right), \max \left(\xi_{1}^{\prime \prime}, \ldots, \xi_{([\sqrt{n}]+1)^{2}}^{\prime \prime}\right)<q_{[\sqrt{n}]+1}\right\} \geqslant 1-\frac{2 \varepsilon}{n},
\end{aligned}
$$


которая при $n \geqslant N$ с учетом (23) дает

$$
\begin{aligned}
\mathbf{P}\left\{R_{n}^{\prime} \mid A_{n}\right\} & \geqslant \mathbf{P}\left\{R_{n}^{\prime} \mid \mu, \nu \geqslant[\sqrt{n}]+1\right\} \mathbf{P}\left\{\mu, \nu \geqslant[\sqrt{n}]+1 \mid A_{n}\right\} \\
& \geqslant\left(1-\frac{2 \varepsilon}{n}\right) \mathbf{P}\{\mu, \nu \geqslant[\sqrt{n}]+1\} \\
& \geqslant\left(1-\frac{2 \varepsilon}{n}\right)\left(1-\frac{\delta}{2 n}\right) \geqslant 1-\frac{2 \varepsilon}{n}-\frac{\delta}{2 n} .
\end{aligned}
$$

В силу малости выбранного выше $\varepsilon$, неравенства (26) и (28) приводят нас к выполнению (20). Лемма 9 доказана.

Требуемая оценка сверху

$$
\sup _{n \in \mathbf{N}} \mathrm{E}_{\theta}\left\{\mathscr{N}\left(G_{n}\right) \mid G_{n}(t) \not \equiv 0\right\} \leqslant 1+\frac{1-|a-b|}{a+b}
$$

в случае $a \geqslant b$ вытекает из следующей леммы. Если же $a<b$, то достаточно вместо $G_{n}(t)$ рассмотреть полином $\left(-G_{n}(t)\right)$.

Лемма 10. Для распределения $\theta_{\delta}$, удовлетворяющего (19) u (20), при всех $n \in \mathbf{N}$ справедливо следующее неравенство:

$$
\mathbf{E}_{\theta_{\delta}}\left\{\mathscr{N}\left(G_{n}\right) \mid G_{n}(t) \not \equiv 0\right\} \leqslant 1+\frac{1-(a-b)}{a+b}+\frac{5}{a^{2}} \delta .
$$

Д ок а з а т ель с т в о. Обозначим $\tau=\tau(n)$ номер максимального по модулю коэффициента из $\xi_{0}, \xi_{1}, \ldots, \xi_{n}$ и воспользуемся введенными в формулировке предыдущей леммы обозначениями событий $R_{n}, R_{n}^{\prime}$ и $A_{n}$. Оценим сначала среднее число корней полинома $G_{n}$ при условии $\xi_{0} \xi_{n} \neq 0$ :

$$
\begin{aligned}
\mathrm{E}\left\{\mathscr{N}\left(G_{n}\right) \mid \xi_{0} \xi_{n} \neq 0\right\}= & \mathbf{E}\left\{\mathscr{N}\left(G_{n}\right) \mid \bar{A}_{n}, \xi_{0} \xi_{n} \neq 0\right\} \mathbf{P}\left\{\bar{A}_{n} \mid \xi_{0} \xi_{n} \neq 0\right\} \\
& +\mathbf{E}\left\{\mathscr{N}\left(G_{n}\right) \mid A_{n}, \xi_{0} \xi_{n} \neq 0\right\} \mathbf{P}\left\{A_{n} \mid \xi_{0} \xi_{n} \neq 0\right\} .
\end{aligned}
$$

Оценим отдельно первое и второе математические ожидания в правой части:

$$
\begin{aligned}
\mathbf{E}\{ & \left.\mathscr{N}\left(G_{n}\right) \mid \bar{A}_{n}, \xi_{0} \xi_{n} \neq 0\right\} \\
= & \mathbf{E}\left\{\mathscr{N}\left(G_{n}\right) \mid R_{n}, \bar{A}_{n}, \xi_{0} \xi_{n} \neq 0\right\} \mathbf{P}\left\{R_{n} \mid \bar{A}_{n}, \xi_{0} \xi_{n} \neq 0\right\} \\
& +\mathbf{E}\left\{\mathscr{N}\left(G_{n}\right) \mid \bar{R}_{n}, \bar{A}_{n}, \xi_{0} \xi_{n} \neq 0\right\} \mathbf{P}\left\{\bar{R}_{n} \mid \bar{A}_{n}, \xi_{0} \xi_{n} \neq 0\right\} .
\end{aligned}
$$

Заметим, что $\left\{\bar{A}_{n}, R_{n}, \xi_{0} \xi_{n} \neq 0\right\} \subset\left\{\xi_{0}, \xi_{n}, \xi_{\tau}<0\right\}$, поэтому, используя следствие к лемме 4 и оценку $\mathscr{N}\left(G_{n}\right) \leqslant n$, получаем

$$
\begin{aligned}
\mathbf{E}\left\{\mathscr{N}\left(G_{n}\right) \mid \bar{A}_{n}, \xi_{0} \xi_{n} \neq 0\right\} \leqslant \mathbf{E}\left\{1-\frac{1}{2}(-1)^{\tau}\left(1+(-1)^{n}\right) \mid R_{n}, \bar{A}_{n}, \xi_{0} \xi_{n} \neq 0\right\} \\
\quad \times \mathbf{P}\left\{R_{n} \mid \bar{A}_{n}, \xi_{0} \xi_{n} \neq 0\right\}+n \mathbf{P}\left\{\bar{R}_{n} \mid \bar{A}_{n}, \xi_{0} \xi_{n} \neq 0\right\} \\
\leqslant\left(1+\frac{1}{2} M_{n}\right)+n \mathbf{P}\left\{\bar{R}_{n} \mid \bar{A}_{n}, \xi_{0} \xi_{n} \neq 0\right\}
\end{aligned}
$$


где

$$
M_{n} \stackrel{\text { def }}{=}-\mathbf{E}\left\{(-1)^{\tau}\left(1+(-1)^{n}\right) \mid R_{n}, \bar{A}_{n}, \xi_{0} \xi_{n} \neq 0\right\} \mathbf{P}\left\{R_{n} \mid \bar{A}_{n}, \xi_{0} \xi_{n} \neq 0\right\} .
$$

Далее, разобьем событие $\left\{\xi_{0} \xi_{n} \neq 0\right\}$ на четыре:

$$
\left\{\xi_{0} \xi_{n} \neq 0\right\}=\Sigma_{1} \cup \Sigma_{2} \cup \Sigma_{3} \cup \Sigma_{4},
$$

где

$$
\begin{array}{ll}
\Sigma_{1} \stackrel{\text { def }}{=}\left\{\xi_{0}>0, \xi_{n}>0\right\}, & \Sigma_{2} \stackrel{\text { def }}{=}\left\{\xi_{0}<0, \xi_{n}<0\right\}, \\
\Sigma_{3} \stackrel{\text { def }}{=}\left\{\xi_{0}>0, \xi_{n}<0\right\}, & \Sigma_{4} \stackrel{\text { def }}{=}\left\{\xi_{0}<0, \xi_{n}>0\right\} .
\end{array}
$$

\section{Имеем}

$$
\begin{aligned}
\mathbf{E}\{ & \left.\mathscr{N}\left(G_{n}\right) \mid A_{n}, \xi_{0} \xi_{n} \neq 0\right\} \\
= & \mathbf{E}\left\{\mathscr{N}\left(G_{n}\right) \mid R_{n}, R_{n}^{\prime}, A_{n}, \Sigma_{1}\right\} \cdot \mathbf{P}\left\{R_{n}, R_{n}^{\prime}, \Sigma_{1} \mid A_{n}, \xi_{0} \xi_{n} \neq 0\right\} \\
& +\mathbf{E}\left\{\mathscr{N}\left(G_{n}\right) \mid R_{n}, R_{n}^{\prime}, A_{n}, \Sigma_{2}\right\} \cdot \mathbf{P}\left\{R_{n}, R_{n}^{\prime}, \Sigma_{2} \mid A_{n}, \xi_{0} \xi_{n} \neq 0\right\} \\
& +\mathbf{E}\left\{\mathscr{N}\left(G_{n}\right) \mid R_{n}, R_{n}^{\prime}, A_{n}, \Sigma_{3}\right\} \cdot \mathbf{P}\left\{R_{n}, R_{n}^{\prime}, \Sigma_{3} \mid A_{n}, \xi_{0} \xi_{n} \neq 0\right\} \\
& +\mathbf{E}\left\{\mathscr{N}\left(G_{n}\right) \mid R_{n}, R_{n}^{\prime}, A_{n}, \Sigma_{4}\right\} \cdot \mathbf{P}\left\{R_{n}, R_{n}^{\prime}, \Sigma_{4} \mid A_{n}, \xi_{0} \xi_{n} \neq 0\right\} \\
& +\mathbf{E}\left\{\mathscr{N}\left(G_{n}\right) \mid \overline{R_{n} \cap R_{n}^{\prime}}, A_{n}, \xi_{0} \xi_{n} \neq 0\right\} \cdot \mathbf{P}\left\{\overline{R_{n} \cap R_{n}^{\prime}} \mid A_{n}, \xi_{0} \xi_{n} \neq 0\right\} .
\end{aligned}
$$

Учитывая, что $\xi_{\tau}>0$ при условии $R_{n}^{\prime}$, используем следствие к лемме 4 для оценки первых четырех слагаемых правой части и неравенство $\mathscr{N}\left(G_{n}\right) \leqslant n$ для оценки пятого слагаемого:

$$
\begin{aligned}
& \mathbf{E}\left\{\mathscr{N}\left(G_{n}\right) \mid A_{n}, \xi_{0} \xi_{n} \neq 0\right\} \\
& \leqslant \mathbf{E}\left\{1-\frac{1}{2}(-1)^{\tau}\left(1+(-1)^{n}\right) \mid R_{n}, R_{n}^{\prime}, A_{n}, \Sigma_{1}\right\} \\
& \times \mathbf{P}\left\{R_{n}, R_{n}^{\prime}, \Sigma_{1} \mid A_{n}, \xi_{0} \xi_{n} \neq 0\right\} \\
& +\mathbf{E}\left\{3+\frac{1}{2}(-1)^{\tau}\left(1+(-1)^{n}\right) \mid R_{n}, R_{n}^{\prime}, A_{n}, \Sigma_{2}\right\} \\
& \times \mathbf{P}\left\{R_{n}, R_{n}^{\prime}, \Sigma_{2} \mid A_{n}, \xi_{0} \xi_{n} \neq 0\right\} \\
& +\mathbf{E}\left\{2-\frac{1}{2}(-1)^{\tau}\left(1-(-1)^{n}\right) \mid R_{n}, R_{n}^{\prime}, A_{n}, \Sigma_{3}\right\} \\
& \times \mathbf{P}\left\{R_{n}, R_{n}^{\prime}, \Sigma_{3} \mid A_{n}, \xi_{0} \xi_{n} \neq 0\right\} \\
& +\mathbf{E}\left\{2+\frac{1}{2}(-1)^{\tau}\left(1-(-1)^{n}\right) \mid R_{n}, R_{n}^{\prime}, A_{n}, \Sigma_{4}\right\} \\
& \times \mathbf{P}\left\{R_{n}, R_{n}^{\prime}, \Sigma_{4} \mid A_{n}, \xi_{0} \xi_{n} \neq 0\right\} \\
& +n \mathbf{P}\left\{\overline{R_{n} \cap R_{n}^{\prime}} \mid A_{n}, \xi_{0} \xi_{n} \neq 0\right\} \\
& =1 \cdot \mathbf{P}\left\{R_{n}, R_{n}^{\prime}, \Sigma_{1} \mid A_{n}, \xi_{0} \xi_{n} \neq 0\right\}+3 \cdot \mathbf{P}\left\{R_{n}, R_{n}^{\prime}, \Sigma_{2} \mid A_{n}, \xi_{0} \xi_{n} \neq 0\right\}
\end{aligned}
$$


$+2 \cdot \mathbf{P}\left\{R_{n}, R_{n}^{\prime}, \Sigma_{3} \mid A_{n}, \xi_{0} \xi_{n} \neq 0\right\}+2 \cdot \mathbf{P}\left\{R_{n}, R_{n}^{\prime}, \Sigma_{4} \mid A_{n}, \xi_{0} \xi_{n} \neq 0\right\}$

$+n \cdot \mathbf{P}\left\{\overline{R_{n} \cap R_{n}^{\prime}} \mid A_{n}, \xi_{0} \xi_{n} \neq 0\right\}+\frac{1}{2} M_{n}^{\prime}$,

где

$$
\begin{aligned}
M_{n}^{\prime} \stackrel{\text { def }}{=} & -\mathbf{E}\left\{(-1)^{\tau}\left(1+(-1)^{n}\right) \mid R_{n}, R_{n}^{\prime}, A_{n}, \Sigma_{1}\right\} \cdot \mathbf{P}\left\{R_{n}, R_{n}^{\prime}, \Sigma_{1} \mid A_{n}, \xi_{0} \xi_{n} \neq 0\right\} \\
& +\mathbf{E}\left\{(-1)^{\tau}\left(1+(-1)^{n}\right) \mid R_{n}, R_{n}^{\prime}, A_{n}, \Sigma_{2}\right\} \cdot \mathbf{P}\left\{R_{n}, R_{n}^{\prime}, \Sigma_{2} \mid A_{n}, \xi_{0} \xi_{n} \neq 0\right\} \\
& -\mathbf{E}\left\{(-1)^{\tau}\left(1-(-1)^{n}\right) \mid R_{n}, R_{n}^{\prime}, A_{n}, \Sigma_{3}\right\} \cdot \mathbf{P}\left\{R_{n}, R_{n}^{\prime}, \Sigma_{3} \mid A_{n}, \xi_{0} \xi_{n} \neq 0\right\} \\
& +\mathbf{E}\left\{(-1)^{\tau}\left(1-(-1)^{n}\right) \mid R_{n}, R_{n}^{\prime}, A_{n}, \Sigma_{4}\right\} \cdot \mathbf{P}\left\{R_{n}, R_{n}^{\prime}, \Sigma_{4} \mid A_{n}, \xi_{0} \xi_{n} \neq 0\right\} .
\end{aligned}
$$

Предложение 2. Выполнены неравенства $M_{n}, M_{n}^{\prime} \leqslant 0$.

Док а зательство п редложения 2. Доказательство первого неравенства будет фактически содержаться в доказательстве второго, коим мы и ограничимся. Из соображений симметрии нетрудно видеть, что для любого события $U$ из $\sigma$-алгебры, порожденной $\xi_{0}, \xi_{n}$, выполнено

$$
\begin{aligned}
\mathbf{P}\left\{\tau=1 \mid R_{n}, R_{n}^{\prime}, A_{n}, U\right\} & =\mathbf{P}\left\{\tau=2 \mid R_{n}, R_{n}^{\prime}, A_{n}, U\right\}=\cdots \\
& =\mathbf{P}\left\{\tau=n-1 \mid R_{n}, R_{n}^{\prime}, A_{n}, U\right\}
\end{aligned}
$$

что влечет

$$
\begin{aligned}
& \mathbf{E}\left\{(-1)^{\tau} \mid R_{n}, R_{n}^{\prime}, A_{n}, U\right\} \\
& =\left\{\begin{aligned}
\mathbf{P}\{\tau=0 \mid & \left.R_{n}, R_{n}^{\prime}, A_{n}, U\right\}+\mathbf{P}\left\{\tau=n \mid R_{n}, R_{n}^{\prime}, A_{n}, U\right\} \\
-\mathbf{P}\left\{\tau=1 \mid R_{n}, R_{n}^{\prime}, A_{n}, U\right\}, & n \text { - четное, } \\
\mathbf{P}\{\tau=0 \mid & \left.R_{n}, R_{n}^{\prime}, A_{n}, U\right\} \\
-\mathbf{P}\left\{\tau=n \mid R_{n}, R_{n}^{\prime}, A_{n}, U\right\}, & n \text { - нечетное. }
\end{aligned}\right.
\end{aligned}
$$

Положим $U=\Sigma_{1}$. Тогда

$$
\begin{aligned}
& \mathbf{P}\left\{\tau=0 \mid R_{n}, R_{n}^{\prime}, A_{n}, \Sigma_{1}\right\}=\mathbf{P}\left\{\tau=n \mid R_{n}, R_{n}^{\prime}, A_{n}, \Sigma_{1}\right\} \\
& \quad \geqslant \mathbf{P}\left\{\tau=n \mid R_{n}, R_{n}^{\prime}, A_{n}, \xi_{0}>0\right\}=\mathbf{P}\left\{\tau=1 \mid R_{n}, R_{n}^{\prime}, A_{n}, \xi_{0}>0\right\} \\
& \quad \geqslant \mathbf{P}\left\{\tau=1 \mid R_{n}, R_{n}^{\prime}, A_{n}, \Sigma_{1}\right\},
\end{aligned}
$$

поэтому при четном $n$

$$
\mathbf{E}\left\{(-1)^{\tau} \mid R_{n}, R_{n}^{\prime}, A_{n}, \Sigma_{1}\right\} \geqslant 0 .
$$

Положим $U=\Sigma_{2}$. Тогда

$$
\mathbf{P}\left\{\tau=0 \mid R_{n}, R_{n}^{\prime}, A_{n}, \Sigma_{2}\right\}=\mathbf{P}\left\{\tau=n \mid R_{n}, R_{n}^{\prime}, A_{n}, \Sigma_{2}\right\}=0,
$$

поэтому при четном $n$

$$
\mathbf{E}\left\{(-1)^{\tau} \mid R_{n}, R_{n}^{\prime}, A_{n}, \Sigma_{2}\right\} \leqslant 0 .
$$


Положим $U=\Sigma_{3}$. Тогда $\mathbf{P}\left\{\tau=n \mid R_{n}, R_{n}^{\prime}, A_{n}, \Sigma_{3}\right\}=0$, поэтому при нечетном $n$

$$
\mathbf{E}\left\{(-1)^{\tau} \mid R_{n}, R_{n}^{\prime}, A_{n}, \Sigma_{3}\right\} \geqslant 0 .
$$

Положим $U=\Sigma_{4}$. Тогда $\mathbf{P}\left\{\tau=0 \mid R_{n}, R_{n}^{\prime}, A_{n}, \Sigma_{4}\right\}=0$, поэтому при нечетном $n$

$$
\mathbf{E}\left\{(-1)^{\tau} \mid R_{n}, R_{n}^{\prime}, A_{n}, \Sigma_{4}\right\} \leqslant 0 .
$$

Объединяя последние четыре неравенства, получаем $M_{n}^{\prime} \leqslant 0$. Предложение 2 доказано.

Продолжим доказательство леммы. Соотношения (30), (31) и (32) с учетом доказанного предложения дают

$$
\begin{aligned}
& \mathbf{E}\left\{\mathscr{N}\left(G_{n}\right) \mid \xi_{0} \xi_{n} \neq 0\right\} \leqslant\left(1+n \cdot \mathbf{P}\left\{\bar{R}_{n} \mid \bar{A}_{n}, \xi_{0} \xi_{n} \neq 0\right\}\right) \mathbf{P}\left\{\bar{A}_{n} \mid \xi_{0} \xi_{n} \neq 0\right\} \\
& +\left(1 \cdot \mathbf{P}\left\{R_{n}, R_{n}^{\prime}, \xi_{0}>0, \xi_{n}>0 \mid A_{n}, \xi_{0} \xi_{n} \neq 0\right\}\right. \\
& +3 \cdot \mathbf{P}\left\{R_{n}, R_{n}^{\prime}, \xi_{0}<0, \xi_{n}<0 \mid A_{n}, \xi_{0} \xi_{n} \neq 0\right\} \\
& +2 \cdot \mathbf{P}\left\{R_{n}, R_{n}^{\prime}, \xi_{0}>0, \xi_{n}<0 \mid A_{n}, \xi_{0} \xi_{n} \neq 0\right\} \\
& +2 \cdot \mathbf{P}\left\{R_{n}, R_{n}^{\prime}, \xi_{0}<0, \xi_{n}>0 \mid A_{n}, \xi_{0} \xi_{n} \neq 0\right\} \\
& \left.+n \cdot \mathbf{P}\left\{\overline{R_{n} \cap R_{n}^{\prime}} \mid A_{n}, \xi_{0} \xi_{n} \neq 0\right\}\right) \cdot \mathbf{P}\left\{A_{n} \mid \xi_{0} \xi_{n} \neq 0\right\} \\
& \leqslant \mathbf{P}\left\{\bar{A}_{n} \mid \xi_{0} \xi_{n} \neq 0\right\}+n \mathbf{P}\left\{\bar{R}_{n}\right\} \\
& +\left(2-\mathbf{P}\left\{R_{n}, R_{n}^{\prime}, \xi_{0}>0, \xi_{n}>0 \mid A_{n}, \xi_{0} \xi_{n} \neq 0\right\}\right. \\
& +\mathbf{P}\left\{R_{n}, R_{n}^{\prime}, \xi_{0}<0, \xi_{n}<0 \mid A_{n}, \xi_{0} \xi_{n} \neq 0\right\} \\
& \left.+n \cdot \mathbf{P}\left\{\overline{R_{n} \cap R_{n}^{\prime}} \mid A_{n}, \xi_{0} \xi_{n} \neq 0\right\}\right) \cdot \mathbf{P}\left\{A_{n} \mid \xi_{0} \xi_{n} \neq 0\right\} .
\end{aligned}
$$

Учитывая тривиальное следствие из (19) и $(20)$ :

а также соотношения

$$
\mathbf{P}\left\{\overline{R_{n} \cap R_{n}^{\prime}} \mid A_{n}, \xi_{0} \xi_{n} \neq 0\right\} \leqslant \frac{2 \delta}{n \mathbf{P}\left\{A_{n}, \xi_{0} \xi_{n} \neq 0\right\}} \leqslant \frac{2 \delta}{n a^{2}},
$$

$$
\begin{aligned}
\mathbf{P} & \left\{R_{n}, R_{n}^{\prime}, \xi_{0}>0, \xi_{n}>0 \mid A_{n}, \xi_{0} \xi_{n} \neq 0\right\} \\
& \geqslant \mathbf{P}\left\{\xi_{0}>0, \xi_{n}>0 \mid A_{n}, \xi_{0} \xi_{n} \neq 0\right\}-\mathbf{P}\left\{\overline{R_{n} \cap R_{n}^{\prime}} \mid A_{n}, \xi_{0} \xi_{n} \neq 0\right\} \\
& \geqslant \mathbf{P}\left\{\xi_{0}>0, \xi_{n}>0 \mid \xi_{0} \xi_{n} \neq 0\right\}-\mathbf{P}\left\{\overline{R_{n} \cap R_{n}^{\prime}} \mid A_{n}, \xi_{0} \xi_{n} \neq 0\right\} \\
& =\frac{a^{2}}{(a+b)^{2}}-\mathbf{P}\left\{\overline{R_{n} \cap R_{n}^{\prime}} \mid A_{n}, \xi_{0} \xi_{n} \neq 0\right\}
\end{aligned}
$$

и

$$
\begin{aligned}
& \mathbf{P}\left\{R_{n}, R_{n}^{\prime}, \xi_{0}<0, \xi_{n}<0 \mid A_{n}, \xi_{0} \xi_{n} \neq 0\right\} \\
& \quad \leqslant \mathbf{P}\left\{\xi_{0}<0, \xi_{n}<0 \mid A_{n}, \xi_{0} \xi_{n} \neq 0\right\} \\
& \quad \leqslant \mathbf{P}\left\{\xi_{0}<0, \xi_{n}<0 \mid \xi_{0} \xi_{n} \neq 0\right\}=\frac{b^{2}}{(a+b)^{2}},
\end{aligned}
$$


из (33) получаем

$$
\begin{aligned}
\mathbf{E}\left\{\mathscr{N}\left(G_{n}\right) \mid \xi_{0} \xi_{n} \neq 0\right\} & \leqslant 2-\frac{a^{2}}{(a+b)^{2}}+\frac{b^{2}}{(a+b)^{2}}+\delta+\frac{2 \delta}{a^{2}}+\frac{2 \delta}{n a^{2}} \\
& \leqslant 2-\frac{a-b}{a+b}+\frac{5}{a^{2}} \delta .
\end{aligned}
$$

Теперь можно вывести оценку в общем случае. Напомним, что величины $\zeta_{n}, \eta_{n}$ были введены в конце доказательства леммы 8. Имеем:

$$
\begin{aligned}
\mathbf{E} & \left\{\mathscr{N}\left(G_{n}\right) \mid G_{n}(t) \not \equiv 0\right\} \\
& =\sum_{0 \leqslant i+j \leqslant n} \mathbf{E}\left\{\mathscr{N}\left(G_{n}\right) \mid \zeta_{n}=i, \eta_{n}=j\right\} \mathbf{P}\left\{\zeta_{n}=i, \eta_{n}=j\right\} \\
& \leqslant \sum_{0 \leqslant i, j<\infty}\left(\mathbf{E}\left\{\mathscr{N}\left(G_{n-i-j}\right) \mid \xi_{0} \xi_{n-i-j} \neq 0\right\}+j\right) c^{i+j}(1-c)^{2} .
\end{aligned}
$$

Воспользовавшись (34) и, как и в оценке снизу, соотношениями (17), получаем требуемое неравенство. Лемма 10 доказана.

4. Заключительные замечания. Рассмотрим произвольное подмножество $\Lambda_{0} \subset \Lambda$, где

$$
\Lambda \stackrel{\text { def }}{=}\left\{(a, b) \in \mathbf{R}^{2} \mid 0<a+b \leqslant 1\right\} .
$$

Объединяя соотношения (1) по всевозможным $(a, b) \in \Lambda_{0}$, получим

$$
\inf _{\theta \in \Theta\left(\Lambda_{0}\right)} \sup _{n \in \mathbb{N}} \mathbf{E}_{\theta}\left\{\mathscr{N}\left(G_{n}\right) \mid G_{n}(t) \not \equiv 0\right\}=1+\inf _{(a, b) \in \Lambda_{0}} \frac{1-|a-b|}{a+b},
$$

где $\Theta\left(\Lambda_{0}\right) \stackrel{\text { def }}{=} \bigcup_{(a, b) \in \Lambda_{0}} \Theta_{a, b}$.

П р и м е р 1. Пусть $\Lambda_{0}=\Lambda$. В этом случае $\Theta\left(\Lambda_{0}\right)=\Theta$, и (35) принимает вид

$$
\inf _{\theta \in \Theta} \sup _{n \in \mathbb{N}} \mathbf{E}_{\theta}\left\{\mathscr{N}\left(G_{n}\right) \mid G_{n}(t) \not \equiv 0\right\}=1 .
$$

П р и м е р 2. Если $\Lambda_{0}=\{(a, b) \in \Lambda \mid a+b=1-c\}$, где $0 \leqslant c<1$, то мы получим

$$
\inf _{\theta \in \Theta_{c}} \sup _{n \in \mathbf{N}} \mathbf{E}_{\theta}\left\{\mathscr{N}\left(G_{n}\right) \mid G_{n}(t) \not \equiv 0\right\}=\frac{1}{1-c},
$$

где $\Theta_{c}$ есть семейство всех невырожденных распределений с весом $c$ в начале координат.

П р и м е р 3. Пусть $\Lambda_{0}=\{(a, b) \in \Lambda \mid a=b\}$. Тогда $\Theta\left(\Lambda_{0}\right)=\Theta^{\prime}$ есть семейство всех невырожденных распределений с равными мерами положительной и отрицательной полуосей, и мы имеем

$$
\inf _{\theta \in \Theta^{\prime}} \sup _{n \in \mathbb{N}} \mathbf{E}_{\theta}\left\{\mathscr{N}\left(G_{n}\right) \mid G_{n}(t) \not \equiv 0\right\}=2 .
$$


Покажем, что соотношение (36) можно усилить. Именно, $\inf _{\theta \in \Theta^{*}} \sup _{n \in \mathrm{N}} \mathbf{E}_{\theta}\left\{\mathscr{N}\left(G_{n}\right) \mid G_{n}(t) \not \equiv 0\right\}=2$, где $\Theta^{*}$ обозначает класс всех невырожденных симметричных распределений.

Поскольку $\Theta^{*} \subset \Theta^{\prime}$, достаточно получить оценку сверху. Для этого рассмотрим распределение $\theta_{\delta}$, построенное в лемме 9 , в случае $a=1$. Если его симметрично продолжить на отрицательную полуось и нормировать двойкой до вероятностного, то при этом у случайного полинома коэффициенты домножатся на \pm 1 случайным образом. Так как в условии леммы 4 участвуют лишь абсолютные значения коэффициентов полинома, то полученное таким образом симметричное распределение $\theta_{\delta}^{*}$ по-прежнему будет удовлетворять (19). Следовательно, для него выполнено

$$
\begin{aligned}
\mathbf{E} \mathscr{N}^{+}\left(G_{n}\right) & =\mathbf{E}\left\{\mathscr{N}^{+}\left(G_{n}\right) \mid R_{n}\right\} \mathbf{P}\left\{R_{n}\right\}+\mathbf{E}\left\{\mathscr{N}^{+}\left(G_{n}\right) \mid \overline{R_{n}}\right\} \mathbf{P}\left\{\overline{R_{n}}\right\} \\
& \leqslant \mathbf{E}\left\{\mathscr{N}^{+}\left(G_{n}\right) \mid R_{n}\right\} \mathbf{P}\left\{R_{n}\right\}+\delta .
\end{aligned}
$$

Напомним, что $\tau$ обозначает максимальный по модулю коэффициент. Имеем:

$$
\begin{aligned}
& \mathbf{E}\left\{\mathscr{N}^{+}\left(G_{n}\right) \mid R_{n}\right\} \mathbf{P}\left\{R_{n}\right\} \\
&= \mathbf{E}\left\{\mathscr{N}^{+}\left(G_{n}\right) \mid \xi_{0} \xi_{n}>0, \xi_{0} \xi_{\tau}>0, R_{n}\right\} \mathbf{P}\left\{\xi_{0} \xi_{n}>0, \xi_{0} \xi_{\tau}>0, R_{n}\right\} \\
&+\mathbf{E}\left\{\mathscr{N}^{+}\left(G_{n}\right) \mid \xi_{0} \xi_{n}>0, \xi_{0} \xi_{\tau}<0, R_{n}\right\} \mathbf{P}\left\{\xi_{0} \xi_{n}>0, \xi_{0} \xi_{\tau}<0, R_{n}\right\} \\
&+\mathbf{E}\left\{\mathscr{N}^{+}\left(G_{n}\right) \mid \xi_{0} \xi_{n}<0, R_{n}\right\} \mathbf{P}\left\{\xi_{0} \xi_{n}<0, R_{n}\right\} .
\end{aligned}
$$

Применяя лемму 4, получаем

$$
\begin{aligned}
& \mathbf{E}\left\{\mathscr{N}^{+}\left(G_{n}\right) \mid R_{n}\right\} \mathbf{P}\left\{R_{n}\right\} \leqslant 0 \cdot \mathbf{P}\left\{\xi_{0} \xi_{n}>0, \xi_{0} \xi_{\tau}>0, R_{n}\right\} \\
& \quad+2 \cdot \mathbf{P}\left\{\xi_{0} \xi_{n}>0, \xi_{0} \xi_{\tau}<0, R_{n}\right\}+1 \cdot \mathbf{P}\left\{\xi_{0} \xi_{n}<0, R_{n}\right\} \\
& \leqslant 2 \mathbf{P}\left\{\xi_{0} \xi_{n}>0, \xi_{0} \xi_{\tau}<0\right\}+\mathbf{P}\left\{\xi_{0} \xi_{n}<0\right\} \\
& \quad \leqslant 2 \mathbf{P}\left\{\xi_{0} \xi_{n}>0, \xi_{0} \xi_{1}<0\right\}+\mathbf{P}\left\{\xi_{0} \xi_{n}<0\right\}=2 \cdot \frac{1}{4}+\frac{1}{2}=1
\end{aligned}
$$

что, с учетом (37), дает

$$
\mathbf{E} \mathscr{N}^{+}\left(G_{n}\right) \leqslant 1+\delta
$$

Поскольку для симметричных распределений среднее число положительных корней полинома совпадает со средним числом отрицательных, мы приходим к оценке

$$
\mathbf{E} \mathscr{N}\left(G_{n}\right) \leqslant 2+2 \delta
$$


П р и м е р 4. Если в предыдущем примере к распределению $\theta_{\delta}^{*}$ добавить атом в начале координат (нормировав при этом соответствующим образом), то можно аналогично получить соотношение

$$
\inf _{\theta \in \Theta_{c}^{*}} \sup _{n \in \mathbb{N}} \mathbf{E}_{\theta}\left\{\mathscr{N}\left(G_{n}\right) \mid G_{n}(t) \not \equiv 0\right\}=1+\frac{1}{1-c}, \quad 0 \leqslant c<1,
$$

где $\Theta_{c}^{*}$ обозначает класс всех симметричных распределений с весом $c$ в начале координат.

Авторы благодарны И.А. Ибрагимову за введение их в данную тематику и ряд ценных советов, а также Ю. А. Зайцеву за несколько замечаний, позволивших упростить доказательства некоторых лемм.

Часть работы была выполнена во время пребывания первого автора в Институте математической стохастики (Университет Геттингена), а также в Университете Билефельда. Он благодарит М. Денкера и Ф. Гетце за их гостеприимство.

\section{СПИСОК ЛИТЕРАТУРЫ}

1. Bloch A., Pólya G. On the roots of certain algebraic equations. - Proc. London Math. Soc., 1931, v. 33, p. 102-114.

2. Littlewood J.E., Offord A.C. On the number of real roots of a random algebraic equation. - J. London Math. Soc., 1938, v. 13, p. 288-295.

3. Littlewood J.E., Offord A.C. On the number of real roots of a random algebraic equation. II. - Proc. Cambridge Philos. Soc., 1939, v. 35, p. 133-148.

4. Littlewood J.E., Offord A.C. On the number of real roots of a random algebraic equation. III. - Матем. сб., 1943, т. 12 № 3, с. 277-286.

5. Kac M. On the average number of real roots of a random algebraic equation. - Bull. Amer. Math. Soc., 1943, v. 49, p. 314-320.

6. Kac $M$. On the average number of real roots of a random algebraic equation. II. Proc. London Math. Soc., 1949, v. 50, p. 390-408.

7. Erdös $P$., Offord $A$. On the number of real roots of a random algebraic equation. Proc. London Math. Soc. (3), 1956, v. 6, p. 139-160.

8. Ибрагимов И. А., Маслова Н.Б. Среднее число вещественных корней случайных полиномов. - Докл. АН СССР, 1971, т. 199, с. 13-16.

9. Запорожеи Д. Н. Пример случайного полинома с необычным поведением корней. - Теория вероятн. и ее примен., 2005 , т. 50 , в. 3 , с. 549-555.

10. Прохоров Ю. В., Розанов Ю. А. Теория вероятностей. М.: Наука, 1973, 496 с.

Поступила в редакцию 29.XII. 2007 